\title{
Gilles Gagné
}

sociologue, professeur au département de sociologie, Université Laval

(1983)

\section{"Une science synthétique du réel? (À propos de «La nature de la nature» d'Edgar Morin)”}

\footnotetext{
Un document produit en version numérique par Jean-Marie Tremblay, bénévole, professeur de sociologie au Cégep de Chicoutimi

Courriel: jean-marie tremblay@uqac.ca

Site web pédagogique : http://www.uqac.ca/jmt-sociologue/
}

Dans le cadre de la collection: "Les classiques des sciences sociales"

Site web: http://classiques.uqac.ca/

Une collection développée en collaboration avec la Bibliothèque

Paul-Émile-Boulet de l'Université du Québec à Chicoutimi

Site web: http://bibliotheque.uqac.ca/ 
Cette édition électronique a été réalisée par Jean-Marie Tremblay, bénévole, professeur de sociologie au Cégep de Chicoutimi à partir de :

Gilles Gagné

"Une science synthétique du réel ? (À propos de «La nature de la nature» d'Edgar Morin)”.

Un article publié dans la revue Cahiers de recherche sociologique, vol. 1, septembre 1983, pp. 85-101. Montréal: Département de sociologie, UQÀM. Numéro intitulé: “Connaissance et société”.

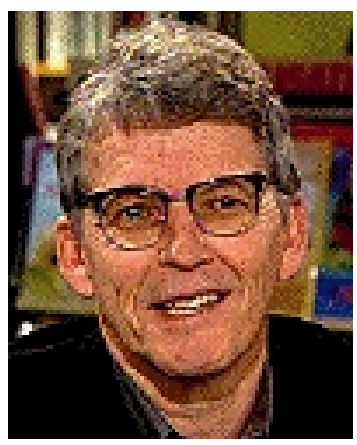

M. Gilles Gagné, sociologue, professeur au département de sociologie de l’Université Laval, nous a accordé le 30 mars 2007 son autorisation de diffuser électroniquement cet article dans Les Classiques des sciences sociales.

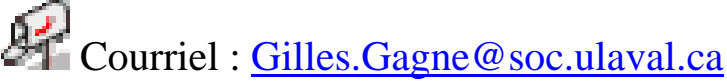

Polices de caractères utilisée :

Pour le texte: Times New Roman, 14 points.

Pour les citations : Times New Roman, 12 points.

Pour les notes de bas de page : Times New Roman, 12 points.

Édition électronique réalisée avec le traitement de textes Microsoft Word 2004 pour Macintosh.

Mise en page sur papier format : LETTRE (US letter), 8.5’’ x 11'’)

Édition numérique réalisée le 30 mars 2007 à Chicoutimi, Ville de Saguenay, province de Québec, Canada. 


\section{Gilles Gagné (1983)}

sociologue, professeur au département de sociologie, Université Laval

\section{"Une science synthétique du réel ? (À propos de «La nature de la nature» d'Edgar Morin)”.}

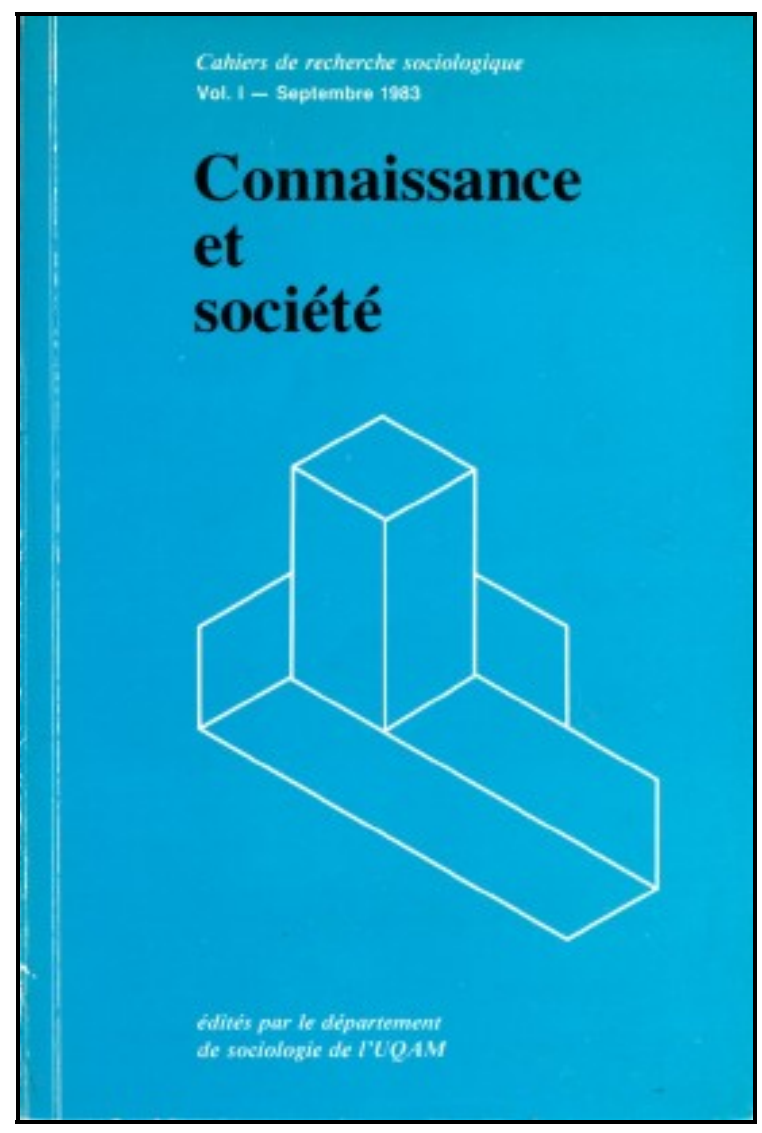

Un article publié dans la revue Cahiers de recherche sociologique, vol. 1, septembre 1983, pp. 85-101. Montréal: Département de sociologie, UQÀM. Numéro intitulé: “Connaissance et société”. 


\section{Table des matières}

1. L'ancienne fondation du savoir...

...et la nouvelle

2. L'unique objet...

... du seul sujet 


\section{Gilles Gagné}

\section{"Une science synthétique du réel ? (À propos de «La nature de la nature» d'Edgar Morin)”.}

Un article publié dans la revue Cahiers de recherche sociologique, vol. 1, septembre 1983, pp. 85-101. Montréal: Département de sociologie, UQÀM. Numéro intitulé: “Connaissance et société”.

\section{L'ancienne fondation du savoir...}

$\underline{\text { Retour à la table des matières }}$

Il est heureux que celui qui a compris «qu'il était sans espoir de seulement réfuter et que seule une nouvelle fondation pouvait ruiner l'ancienne » 1 ne se soit pas mis dans la tête de s'en prendre à l'an-

1 Edgar Morin, La nature de la nature, Éditions du Seuil, Paris, 1977, p. 21. Morin fait partie de ces quelques hommes qui réussissent, depuis une trentaine d'années à chevaucher la crête des vagues qui déconstruisent ---> reconstruisent (excusez ce morinisme) la conscience moderne. Sa qualité première est la sensibilité aux défauts de la force des habitudes disparates qui, dans le domaine des sciences, tendent aujourd'hui à former un « système " qui serait enfin impensable. (Jamais, pourtant, ne le sent-on s'abandonner de gaieté de coeur à la critique de quelque morceau de savoir que ce soit, et bien qu'il ait encore néanmoins plusieurs milliers « d'idées », il ne s'en est pas moins purgé, quand cela fut nécessaire, de plusieurs milliers d'autres dans le passé). Notre époque, c'est évident, Morin la sent dans son ventre et s'il a accouché jusqu'à date de plusieurs Morin différents, il n'a encore accouché d'aucun Morin à contretemps. (Il ouvre la bouche et c'est, en direct, son temps qui parle ; mais dès qu'il a parlé, il sent que ça a déparlé (c'est le « ricanement interne » qui l'en avertit) ; habituellement, du moins c'est ce qu'il dit, il entre d'abord en crise mais ensuite conçoit à nouveau du neuf). Morin est fertile.

Son défaut premier, depuis La nature de la nature, ce sont les mots qu'il s'est mis à fournir à son ventre : il les sélectionne dorénavant un par un, soigneusement, en fait de petits paquets en en reliant quelques-uns par des flèches et consacre à chaque paquet un chapitre. C'est de ce défaut qu'il est question ici, et non pas des qualités susmentionnées. Évidemment, on dira que les 
cienne fondation en tant que telle mais seulement à son ancienneté car alors il eût manifestement été contraint de passer par la réfutation et par tout ce qui s'ensuit le plus souvent. Pour ruiner la fondation considérée indépendamment de son âge, en effet, il faut discuter sérieusement avec elle et prendre le risque de lui trouver des avantages alors que pour ce qui est des vieilleries, il y a des préjugés qui peuvent en disposer avant cinq heures pourvu qu'elles leur soient proprement désignées en tant que telles. S'agissant de l'ancienne fondation du savoir cependant, il ne faut pas se réjouir trop vite de la banqueroute dont elle se trouverait menacée par la simple entrée en scène d'une nouvelle fondation qui, quant à elle et avant même d'avoir trouvé bailleur, annonce qu'elle désespère de réfuter la victime et qu'elle entend donc commencer par la ruiner. Si « seule une nouvelle fondation peut ruiner l'ancienne », c'est peut-être que la nouvelle croit comprendre que les arguments qu'elle est en mesure d'invoquer contre l'ancienne seraient d'un bien médiocre poids dans la balance de la rivalité mimétique et qu'elle doit s'en remettre totalement au prestige de sa propre nouveauté pour culbuter son alter ego.

En sciences humaines, en effet, là où il est permis à tout ce qui arrive à se donner pour nouveau d'entrevoir pour soi la destinée la plus haute, il n'est pas rare de voir surgir de telles fondations, charpentes ou aménagements intérieurs qui, en se donnant tout juste la peine de les porter disparues, parviennent à occuper la place de constructions qui étaient encore raisonnablement utiles. Ces nouveautés, le plus souvent, réussissent si bien à attirer sur elles l'attention qui devrait plutôt se porter à ce qu'elles élident, négligent, taisent sciemment et remplacent qu'elles soupçonnent avec finesse qu'il ne se trouvera personne pour les reconnaître elles-mêmes comme nouvelles synthèses, nouvelles fondations, nouveaux paradigmes ou comme recommence-

ouvrages de Morin sont l'ensemble de leurs défauts ---><--qualités. Peut-être, mais c'est cela leur défaut : parti pour un grand voyage d'instruction, Morin rencontre d'abord des gens qui appellent un chat un chien puis d'autres qui appellent un chien un chat ; comme cela le trouble, il concocte alors une synthèse et propose d'appeler un chat ---> chien un chat ---> chien. La procédure est impeccable à ceci près qu'elle consiste à faire de deux clartés locales une confusion impériale. L'aberration pouvant être cohérente, Morin pourrait sans doute faire valoir qu'ainsi il contribue à «dynamiser » les idées en y mettant de l'ordre --> désordre ; et cela est très vrai ---> faux. 
ment de la science ; aussi existe-t-il plusieurs domaines du savoir, aujourd'hui, où les révolutions scientifiques ont la prudence de s'intituler elles-mêmes des révolutions scientifiques et où le moindre discours trouve le moyen de se déblayer un "espace " à révolutionner librement avant même d'avoir fait son premier tour. À la fin, tout s'annonce comme nouvelle approche ou comme anti-méthode et le point de non-retour est atteint lorsque les nouveautés peuvent se ruiner les unes les autres sans avoir à se réfuter. Rien ne bornant plus alors le marché des valeurs spéculatives, il s'emballe et menace constamment de tomber sous l'autorité de ceux qui maîtrisent le mieux l'art de faire passer des vessies pour des lanternes ; l'heure de la concurrence a sonné et bien que tout prétende au prestige des antiques espèces, celles-ci ne sont plus qu'un souvenir trébuchant dans la conscience de ceux qui s'adonnent aux progrès scientifiques du jour.

Les nouvelles fondations du savoir n'ont plus à dire ce qu'elles tiennent pour en être d'anciennes ; mieux, elles n'ont pas même à se préoccuper de savoir de quoi ont bien pu avoir l'air ces vénérables fantasmagories. On aurait bien aimé, par exemple et pour parler comme l'esprit d'objectivité lorsqu'il ne veut pas intervenir en son nom personnel, que les proposeurs d'une nouvelle fondation pour le savoir se soient appliqués à ciseler pour le commun un résumé de l'ancienne fondation telle qu'elle était à la fin de son dernier exercice et qu'ils l'aient fait d'une manière cohérente et précise, conformément à toutes les exigences didactiques. Il est assez étrange d'entendre parler pour la première fois d'une chose aussi importante le jour de sa déchéance : la voilà en un seul paragraphe à la fois baptisée, excommuniée et oubliée. De la même manière, il y a une Nouvelle Synthèse qui bat actuellement la campagne et qui, paradoxalement, « réanalyse » toutes choses sous le soleil sur la base de principes nouveaux; or, personne ne semble avoir la moindre idée de ce qu'était l'Ancienne Synthèse et du haut de ces nouvelles connaissances, nous sommes réduits à faire des suppositions sur la nature de notre ancienne ignorance : nous ne savons pas ce que nous ignorions. Cependant, puisque le premier postulat de la Nouvelle Synthèse stipule que les organismes sont des mécanismes grâce auxquels les gènes se reproduisent et puisque le sens commun, tel qu'il subsiste dans certaines régions, s'imagine que les gènes sont des machins grâce auxquels les organismes se reproduisent, il est probable que les synthétiseurs se soient contentés de faire 
subir au sens commun une rotation de 180 degrés par rapport à la verticale et lui aient trouvé, sous cet angle et selon la méthode éprouvée, une physionomie de nouveau paradigme. C'est donc sans doute le sens commun qui fait ici office d'Ancienne Synthèse et les cégépiens n'ont pas tort d'appeler « esprit de bottine » le sens commun renversé ; cela donne une idée de la grosseur des sabots que chaussent couramment ceux qui font de grands pas en avant dans les hauteurs de la pensée.

Nous avons peut-être affaire à une ronde infernale où il faudrait voir que l'inflation généralisée qui afflige la "production » intellectuelle oblige les recommencements de la science à s'affecter euxmêmes d'un haut coefficient de nouveauté simplement pour ne pas être victimes du préjugé qui détourne le consommateur de tout ce qu'il croit avoir déjà vu et qu'à cette fin ils doivent s'inventer des prédécesseurs à qui faire tenir le rôle d'un ancien savoir global et positif ayant été jadis ce qu'ils aspirent à être maintenant. Condamnés à collaborer avec une manie qu'ils essaient de circonvenir, les intellectuels se verraient alors engagés sur la pente du cynisme, sans autres issues que de devoir emballer leurs apports à la science de la même manière que les Sex Pistols ou Ponce Pilate et ses Lavabos ont emballé leur contribution à la culture populaire. Vaines plaisanteries ? Nous sommes encore loin de là ? Très certainement. Cependant, il n'est pas certain que les garde-fous soient aussi sains qu'on les imagine.

Lorsque l'on renonce à réfuter ce qu'on donne pour faux, que l'on se propose tout bonnement de substituer un savoir à un autre et que l'on a toutes les raisons de croire que personne ne se formalisera de cette anti-méthode, on renonce à quelque chose d'absolument essentiel à cette fameuse science à laquelle souscrit avec tant d'empressement le moindre charabia. Si jamais la science tint à quelque chose, en effet, il semble que ce fut justement à ceci : ce qui n'est pas réfuté ne peut pas être ruiné. Même en élargissant les perspectives et en admettant qu'en sciences humaines c'est de théories et de problématiques globales qu'il s'agit parfois et que ces choses ne sont pas décidables à coup de contre-exemples et d'hypothèses falsifiées, le passage par la discussion ne s'en trouve pas rabaissé au rang d'ornement mais élevé au rang de condition nécessaire. En sciences humaines, précisément, la communication avec le savoir des autres est si fondamentale que les correcteurs d'épreuves devraient être investis du pouvoir de déclarer im- 
propre à la publication tout ce qui prétend avoir des choses si radicalement nouvelles à dire qu'elles rendent inutile l'effort d'en convaincre ceux qui savent autre chose. En sciences humaines, encore, évoquer ou poser d'anciennes fondations et d'anciens paradigmes c'est désigner déjà les tous premiers interlocuteurs qui se tiennent sur la voie des nouveaux, et ces derniers ne peuvent prétendre accéder à aucun objet ni à aucune réalité sans avoir d'abord passé par ce qui est le savoir de ces choses, tel qu'ils le trouvent devant eux. Celui qui dit : " J'ai découvert combien il est vain de ne polémiquer que contre l'erreur : celle-ci renaît sans cesse de principes de pensée qui, eux, se trouvent hors conscience polémique " 2 se dérobe à la première occasion et veut faire l'économie du commencement. En science comme il le dit, et en sciences humaines à plus forte raison, il faut commencer par introduire dans le champ de conscience (polémique ou non) ce qui est sous-jacent - les « principes de pensée » mais aussi les intérêts, la valorisation et les habitudes - à ce que l'on a cru devoir désigner par ailleurs comme erreur. Déclarer que la polémique avec l'erreur est inutile parce que celle-ci dépend de principes de pensée qui sont hors conscience revient à poser que le but de la polémique est ce qui la rend impossible ; autant dire qu'il est inutile de parler parce que les autres ne savent pas déjà ce que l'on va dire et qu'en conséquence il est plus profitable de se détourner d'eux et de monologuer jusqu'à ce qu'ils le découvrent par eux-mêmes. Et c'est exactement ce que se propose le nouveau fondateur : ayant renoncé à réfuter ce qu'il appelle l'erreur, à lui servir des preuves et à argumenter pour lui faire prendre conscience d'elle-même, il se détourne d'elle, l'abandonne aux principes dont elle sourd et entreprend d'accoucher d'une scienza nuova. Mais la question se pose : qui entendra cette déesse, quels seront ses sujets ? Ceux qui n'avaient même rien à voir avec la scienza antica et qui attendaient sans doute la scienza nuova pour devenir savants, ou ceux qui vivent dans la science du passé et qui, malheureusement, n'ont pas encore été « détrompés » ? Si c'est à ces derniers que l'on pense, s'attend-on à ce qu'ils découvrent tout d'un coup ce qu'on ne leur a pas dit parce qu'ils ne le savaient pas ? Bref, comment espère-t-on faire entendre une nouvelle science si l'on commence par poser comme irréalisable la tâche de simplement critiquer l'ancienne et comme irrécupé-

\footnotetext{
2 Ibidem.
} 
rables les résultats auxquels elle est arrivée ? Par un coup de publicité, peut-être?

En fait, ce genre de paradoxe vient sans doute de ce qu'en sciences humaines on est d'autant plus prompt à faire rupture, à changer de terrain, à bouleverser la science et à ruiner les anciennes fondations que l'on a une idée rabougrie de la manière dont ces choses se passent dans les sciences de la nature. Un peu comme si l'histoire des sciences ne servait absolument à rien, on pose que l'erreur admet la vérité seulement si elle lui est assenée du haut d'un nouveau paradigme et que pour polémiquer utilement avec l'ancienne fondation et avoir quelque espoir de l'obliger à reconnaître les arguments qui la troublent, il faut d'abord avoir mis un point final à la nouvelle. L'histoire des vraies sciences enseignerait, somme toute, qu'il n'y a pas de communication possible entre les états successifs d'une science et que les arguments que les "practitioners » s'échangent sans les comprendre ont seulement pour but de tuer le temps nécessaire à l'extinction de la génération dont les erreurs relèvent de "principes de pensée » qui sont " hors conscience polémique ». Et dans ce qu'il reste de l'admirable science ainsi révisée ne figure plus ce pourquoi elle voulait être admirée du temps qu'elle était cela où l'erreur pouvait devenir visible selon ses propres critères et où il n'était pas nécessaire d'être déjà dans le vrai pour faire dégorger au faux les alternatives qu'il réprime. Bref, la conception concurrentielle des sciences est parvenue à réécrire l'histoire de celles-ci de manière à offrir aux sciences humaines un modèle de révolution selon lequel ce n'est pas de la communication d'arguments bien construits que se dégagent parfois des constructions théoriques capables de faire partiellement échec à la divergence des intérêts et de tenir entre parenthèses la multiplicité éclatée des valeurs, mais selon lequel au contraire, un nouveau paradigme sorti de quelque manche par génération spontanée doit d'abord causer la ruine de l'ancien en s'emparant de son marché. Il n'y aurait, en définitive, que la polémique posthume pour offrir à la grande raison la garantie de son triomphe et pour fournir à la discussion les moyens de rompre le mur de «l'incommensurabilité des paradigmes ». Évidemment, pour soutenir qu'une seule des prodigieuses révolutions scientifiques dont on se fait tant de vapeurs se soit déroulée selon ce schéma, il faut surtout de l'audace ; pour ce qui est de réussir une révolution scientifique en prenant cela comme modèle, il faut, en particulier en sciences humai- 
nes, surtout des amis, car la valeur de ces sciences dépend d'autant plus de leur aptitude à être partagées qu'elles visent à rendre pensable ce en quoi chacun a une part. Aussi, lorsque l'on est assez généreux pour supposer à ses contemporains des principes de pensée, on est lié par cette générosité et contraint d'admettre qu'il faudra commencer par parler depuis ce qui est connu. Bien sûr, il faut pour cela être soimême convaincu que l'hypothétique ancien savoir n'est pas clos et que toutes les erreurs qu'il ne réussirait plus à cacher sont autant d'oreilles qui prouvent qu'il n'est pas sourd ; mais, comme c'est une science déjà finie que les recommenceurs sentent grouiller en leur sein, comme ils pensent que le nouveau paradigme doit être radical et qu'ils se proposent de coincer leurs interlocuteurs entre l'adhésion inconditionnelle (s'ils sont de la classe ou de la génération dont l'avènement est espéré) ou le rejet en bloc (s'ils sont de la classe ou de la génération dont la disparition est attendue), il est sans doute normal qu'ils aient imaginé à ce dont ils se savent porteurs des prédécesseurs du même profil, fût-ce en négatif, c'est-à-dire des espèces de bêtises circulaires et parfaites, construites sur des bases irrémédiablement hors conscience. Or, rien ne peut être de cette nature hormis quelques graves maladies mentales et il est permis de croire que les connaissances qui ont résisté aux recommencements radicaux sont loin d'être aussi sérieusement atteintes. Si nous considérons de plus qu'il nous est maintenant proposé de les échanger en vrac et une fois pour toutes contre une mode selon laquelle le calendrier des disciplines devrait être remis à l'année zéro à tous les six mois, nous devenons du coup un peu moins sympathiques à cette entreprise habillée de métaphores coperniciennes et consistant à remplacer les eunuques par les hermaphrodites un jour et à faire l'inverse le lendemain ; car si d'un changement de terrain à l'autre nous devons nous élever jusqu'à l'immobilité de la confusion totale, autant faire sur celui où nous sommes déjà quelques pas en direction d'un éclaircissement partiel.

\section{... et la nouvelle}

Retour à la table des matières

Malheureusement, s'il existe, selon Lorentz, un grand parlement des instincts, il n'existe pas de chambre basse où faire valoir la nécessité d'une suspension temporaire des révolutions scientifiques en 
sciences humaines, en particulier à un moment où les différents discours font tant d'efforts pour appliquer leur commune théorie du discours, c'est-à-dire pour se rendre incommensurables entre eux. Il faut donc s'attendre à ce que ceux qui ont de grandes choses en vue continuent d'éliminer de leur langage tout ce qui est susceptible de communiquer avec le domaine pré-révolutionnaire qu'ils se sont choisi. Dans le cas type, le savant, pour arriver à cela, doit d'abord traverser une longue et obscure période de désapprentissage systématique. Il doit tout désapprendre jusqu'à la racine et arriver ultimement à "remettre en question les bases mêmes de (son) système de pensée ». Durant la traversée de ce désert, la paresse parfois s'empare de lui et il lui arrive de se laisser bercer par l'illusion d'être parvenu à la suprême remise en question, par l'illusion de goûter au repos de l'ignorance enfin naïve. Mais non, il lui faut inlassablement se remettre en route et continuer de désapprendre tout ce qu'il lui reste encore à désapprendre. Invariablement déclenché par les secousses de '68 ou par quelque autre dérèglement du cosmos, cet énorme travail de purge, bien qu'il ne soit encore qu'une petite partie de l'effort de recommencement, donne une première idée de l'étendue du savoir dont fut capable le recommenceur dans le passé et donne ainsi déjà l'ordre de grandeur de celui dont il sera présumément capable dans l'avenir. Il faut remarquer ici que le risque qu'il prend de devenir irréversiblement ignorant est plus que compensé par le fait que toute l'énergie par lui investie dans le désapprentissage préalable de l'ancien savoir viendra grossir la valeur totale de l'éventuelle nouvelle fondation ; si bien que le recommenceur, s'il a le nerf et le crédit qu'il faut pour jouer gros, a avantage à faire d'abord un effort maximum en direction de l'ignorance. Si, dans son élan, il désapprend des choses qu'il n'a jamais sues, tant mieux ; personne n'en saura jamais rien et ceux qui trouvent qu'il s'agit là d'une étrange manière de grossir un investissement sont de toutes manières déjà perdus pour la science qui se prépare.

Au terme de cette régression, en effet, et à toutes fins pratiques, intriquée à elle, s'amorce la reconstruction. Comme tout se joue à la racine, c'est du fondement de la base qu'il faut ici repartir car, nous est-il dit, tout n'est pas de désapprendre : il faut aussi « réapprendre à apprendre ». Évidemment l'affaire est délicate et pour quelqu'un qui est en train de désapprendre jusqu'à son ancienne façon d'apprendre, apprendre à nouveau, fût-ce à apprendre, pose des problèmes que l'invo- 
cation d'un feed-back interrogatif et critique suffit à peine à résoudre. Comment réapprendre à apprendre lorsque l'ancienne manière d'apprendre ne permet plus d'apprendre rien de neuf et que l'on a même désappris ce que l'on avait appris par son office ? C'est un problème presque sans fond et si ceux qui le posent ne s'y trouvent pas définitivement enfermés, ce n'est pas faute d'essayer. Pour sortir de ce cercle, il suffit en fait de continuer à creuser et de découvrir qu'il faut « réorganiser notre système mental pour réapprendre à apprendre ». De là, finalement, il ne reste plus qu'à descendre encore de quelques strates, jusqu'au problème de la réorganisation de l'organisation mentale: « Nous nous servons de notre structure de pensée pour penser. Il nous faudra aussi nous servir de notre pensée pour repenser notre structure de pensée. (...) Sinon, la structure morte continuera à sécréter des pensées pétrifiantes ${ }^{3}$. Se débarrasser de la structure pétrifiante en s'en servant pour la repenser n'étant pas une opération à la portée du commun, contentons-nous d'observer que c'est ici que le chemin du ruminant se sépare de celui du penseur : seul ce dernier en effet connaît le pouvoir déclaratoire de la pensée, c'est-à-dire le pouvoir de se débarrasser des cercles vicieux dont il accouche en les déclarant morts. Ainsi, après s'être tourné à quelques reprises la «structure pétrifiante » entre les dents et l'avoir trouvée coriace et pleine de vie circulaire, il la crache au loin et se retourne pour conclure : c'est ainsi, ditil, qu'il faut se débarrasser de la structure morte ! C'est donc sur cette déclaration que se termine la période de la réorganisation de l'organisation mentale et que s'ouvre celle du réapprentissage de l'apprentissage.

Sur cette route enfin libre, il rencontre d'abord son Socrate électronique ; ensuite, différents Hume le sortent de différents sommeils, il remet sur leurs pieds quelques dialectiques, il résout des énigmes, tranche moult noeuds gordiens et fait divers grands pas en avant. Souvent, cependant, pour un seul grand pas en avant dans la mauvaise direction, il doit revenir loin derrière : ayant remis en question ses prémisses psychologiques, il se lance en avant mais seulement pour se découvrir bientôt prisonnier de prémisses anthropologiques encore plus pétrifiantes. Malgré tout, il se dégage à nouveau et sombre à nouveau, comme il le découvre plus tard, dans un délire socio-culturel ou

3 Ibidem. 
physico-chimique qui ne valent guère mieux. À chaque détour, à chaque chute, à chaque triomphe correspondent des départs, des arrivées, des endroits et des gens : c'est grâce à tel théoricien qui l'a accueilli à sa descente d'avion qu'il a fait la connaissance d'une femme géniale à qui il doit tout puisqu'elle lui a passé un bouquin qui lui a ouvert les yeux. Et ainsi, brin par brin, un grand voyage dans le savoir se tisse de centaines de voyages terrestres entre des Instituts et des Centres où ceux qui s'y trouvent ont des chances égales de déclencher chez le recommenceur un des nombreux recommencements secondaires qui s'enchâssent habituellement dans un recommencement majeur. Chaque articulation de la doctrine en voie d'élaboration évoque la personne qui a induit la remise en question qui l'a rendue possible et chaque personne occupe une place donnée sur un registre climatologique extrêmement étendu : l'auteur a bien aimé réfléchir aux postulats de la théorie générale des systèmes en compagnie de A... sous le ciel limpide d'une île grecque avant d'aller vérifier quelques détails à la bibliothèque de l'Université McGill. (Nous en savons des choses !) À la fin, le fichier de référence de la nouvelle fondation a l'allure d'un carnet d'adresses où les perpétuelles migrations des pourvoyeurs d'idées et des déclencheurs de synthèses n'ont laissé que ratures de ratures ; voilà pourquoi il ne lui reste plus alors qu'à se " contraindre à la solitude du pionnier » et à se " brancher sur le patrimoine planétaire » car le fondateur, s'il sait qu'il a les adresses de toutes ses connaissances, n'en déchiffre plus aucune.

Soudainement donc, il atteint ce point à partir d'où il n'y a plus qu'à raturer des ratures et à recommencer de vieux recommencements. La pensée qu'il élabore à mesure qu'il avance, travaillée en profondeur par l'habitude du désapprentissage, ne résiste plus à rien ; le moindre grain de sable dans la soupe planétaire lui broie les dents et la plus insignifiante découverte dans la science de l'eau chaude soulève des raz de marée qui engloutissent tout ce qui était acquis. Elle trébuche à chaque pas sur des détails sur la base desquels il lui semble que tout devrait être " réanalysé » et, voulant additionner ce qui est hétérogène, elle tricote ensemble le trivial et le quelconque mais laisse chroniquement filer toutes les mailles de l'ouvrage pour embrocher quelques mystères. C'est une pensée qui juge tout à partir de sa propre tendance à l'effritement et tout ce qui lui vaut quelque chose lui vaut une remise en question de ses fondements. Lui fait-on l'honneur de lui offrir le 
manuscrit de Le hasard et la nécessité ? Elle rend la politesse en profitant de l'occasion pour s'offrir une "reconversion théorique » majeure. Rendue à ce point, elle est une pensée dont toutes les modalités de développement se ramènent à la conversion, à la reconversion et à la re-reconversion.

Ce sont là quelques-unes des raisons pour lesquelles le recommenceur, un beau matin, s'isole dans un poêle et, à l'abri des influences, donne sa forme définitive - mais parfaitement arbitraire de son propre point de vue - à la doctrine pour laquelle il a fallu tout rompre ce qui a pu être imaginé de barrières. Dans le cas idéal, la nouvelle doctrine aura le bonheur de faire reposer le monde sur un principe minuscule auquel il lui suffira ensuite de se conformer elle-même pour en faire la preuve : posant d'abord "le désordre organisateur », le fondateur pourra faire un bilan aussi désordonné qu'il lui plaît des innombrables conséquences de ce principe et laisser les tendances autoréorganisatrices de ce magma produire la synthèse dont le présume capable le principe initial. Car la pensée n'est plus tant une construction et un résultat qu'un processus immanent au vocabulaire. À la limite, elle est une vallée vers où un entonnoir de mots fait tout converger ; et ce qui passe par elle, elle ne l'intègre pas, elle ne le digère pas, elle ne "l'organise " pas : elle en fait du baby food. Les quartiers de viande et les domaines de cohérence qu'elle engouffre, les discours qui ont encore un sens et les légumes à peu près reconnaissables qu'elle se tape, elle en fait une pâte lisse et onctueuse, une pâte universelle et catholique dont les molécules en désordre s'auto-réorganisent au gré des récipients où elles tombent dans le public. Au total, cela fait une nouvelle synthèse de plus, elle aussi conforme à la science et adaptée aux masses ; c'est un effort de pensée qui demande à être apprécié selon le poids total des matériaux qui ont été pulvérisés ensemble. 


\section{L'unique objet...}

$\underline{\text { Retour à la table des matières }}$

La sorte de synthèse qu'ont en vue les recommenceurs n'est cependant pas étrangère à une forme de tension vers l'unité dont il est difficile de faire abstraction : nous voulons le monde comme totalité et s'il se donne comme désordre et contradiction, nous voulons que la théorie s'approprie, fût-ce d'une manière désordonnée et contradictoire, tout le désordre et toutes les contradictions. Or, il arrive que les changeurs de terrain, qui sont à l'écoute de la confusion d'une oreille et de l'aspiration à l'unité de l'autre, trouvent que la confusion est universelle, ce qui est exagéré, et que l'aspiration à l'unité doit absolument commander la réflexion, ce qui reste un point de vue tant que l'on n'a pas déterminé à quel plan cette aspiration peut légitimement être tenue pour impérative. Se lançant alors sur la trace de toute vérité qui soit propre à rassurer, ils sont satisfaits si à la fin ils peuvent au moins démontrer que la bouillie forme un tout : tout est matériel, tout est biologique, tout est économique ou, lorsqu'on ne veut s'aliéner aucune corporation, tout est physico-bio-anthropo-socio-économique. L'inquiétude n'en demandait pas tant.

La science du réel se caractérise donc d'abord par son rapport à ce qui s'exprime comme besoin d'une synthèse et à ce chapitre il est étonnant qu'au lieu de commencer par examiner, comprendre et critiquer ce besoin, elle se soit empressée d'y répondre comme à une demande parfaitement solvable. Il est possible, après tout, que nous voulions parfois des choses absurdes et il est étrange que même lorsque nous lui avons demandé de faire tenir ensemble la totalité des certitudes, (alors que nous confessions honnêtement n'en avoir plus aucune qui concerne la totalité) la science des recommenceurs nous ait trouvés raisonnables et se soit promptement mise en route. Voici d'ailleurs de quelle oreille le synthétiseur moyen entend le manque qu'il ambitionne de combler :

« Tout néophyte entrant dans la Recherche se voit imposer un renoncement majeur à la connaissance. On le convainc que l'époque des Pic de la 
Mirandole est révolue depuis trois siècles, qu'il est désormais impossible de se constituer une vision et de l'homme et du monde. (...) On lui démontre que l'accroissement et l'hétérogénéisation du savoir dépassent toutes possibilités d'engrammation et de traitement par le cerveau humain. On lui assure qu'il faut non le déplorer, mais s'en féliciter. (...) Désormais spécialiste, le chercheur se voit offrir la possession exclusive d'un fragment du puzzle dont la vision globale doit échapper à tous et à chacun ${ }^{4}$. »

Évidemment, et à moins de faire un effort délibéré dans le sens contraire, chacun reconnaît cette nostalgie d'entrée de jeu : Ah ! Que ne se trouve-t-il quelque Pic de la Mirandole, nous est-il tous arrivé de soupirer, pour articuler ensemble les morceaux éparpillés du savoir tel que se le partagent les spécialités et s'élever ainsi à la connaissance du monde ! Sans doute cette aspiration à la synthèse est-elle légitime en elle-même et sans doute fait-elle honneur à ceux qui s'en montrent capables; sans doute aussi est-elle assez générale pour indiquer un problème qui ne peut pas être négligé mais tout cela est secondaire ici. Ce qui importe c'est que ce besoin, tel que le recueille nos serviteurs dans la pensée, est déjà l'opération de s'enfermer dans le positivisme et que tout effort visant à le satisfaire dans cette forme doit se révéler comme tentative de saturer l'espace positiviste, comme volonté d'en boucher les pores et d'en combler les vides, bref, comme prétention de " résoudre », dans cet espace-là, le problème de la synthèse et d'en rendre inutile toute formulation alternative.

Passons rapidement sur le désir sincère du synthétiseur d'être le Pic de la Mirandole de notre siècle : pour être à la hauteur de son modèle, en effet, illustre pour avoir prouvé que toutes les religions adoraient le même Dieu, il lui faut aujourd'hui faire la preuve que « la physis est l'unique objet de la science » ${ }^{5}$; passons aussi sur cette remarque profonde concernant les capacités d'engrangeage du cerveau: comme « l'organe le plus complexe de l'univers », selon celui-ci, est l'organe favori du "matérialisme de notre époque », comme la puissance $\left(10^{14}\right)$ de ce " chef-d'oeuvre de l'évolution », selon celui-là, fait flé-

4 Idem, p. 12.

5 « Il faut aussi une théorie - une pensée - transdisciplinaire qui s'efforce d'embrasser l'objet, l'unique objet, à la fois continu et discontinu, de la science : la physis ». Edgar Morin, Le paradigme perdu : La nature humaine, Éditions du Seuil, Paris, 1973, p. 229. 
chir plus d'une modeste paire de genoux $\left(2^{1}\right)$ et comme la science synthétique du réel est l'effort de nous doter, sous la forme d'une Physiologie du Cerveau de la Phénoménologie de l'Esprit qui nous convienne, chacun a amplement entendu parler de cette grande chose et de son rôle dans l'auto-développement de la physis. Venons-en plutôt à ce qui est le plus standard dans l'exposé qui nous est fait du mal du siècle, c'est-à-dire venons-en au manque d'une vision du monde et à l'impitoyable Recherche qui interdit à ses agents de s'élever audessus du puzzle pour en prendre une vision globale : la Recherche, conformément à sa logique interne, assigne à chacun sa branche et sa branche lui cache l'arbre ; or, comme l'arbre est le monde il n'est plus possible à l'habitant d'une branche de se faire une idée du monde ; le seul espoir, alors, c'est qu'un citoyen de la Recherche, sans renoncer à la dignité dont elle l'a revêtu, prenne sur lui d'en bafouer en secret les canons et entreprenne de passer par toutes les branches et de descendre ensuite jusqu'au tronc pour saisir enfin la profonde unité de la science (donc du monde), c'est-à-dire pour réconcilier la connaissance du réel avec elle-même en la totalisant.

Voilà en quelque sorte le " Go west young men », l'épopée de l'intellection positive : les spécialités se sont tellement multipliées, tant de sciences isolées accumulent tant de connaissances qui ne communiquent plus entre elles, tant de nouveaux domaines, tant de nouvelles méthodes, tant de nouveaux faits s'ajoutent chaque jour au bagage déjà colossal qui s'est accumulé depuis des siècles qu'il nous faut quelqu'un qui soit capable, dans le cadre d'une stratégie globale, de tout engranger ce qui est crucial et puisse en conséquence nous dire de quoi a l'air le monde. Comme le dit la chanson : le marché est là, les capitaux sont là, le besoin a déjà défini la marchandise qu'il lui faut ; qui inventera la formule d'une science synthétique du réel qui pourra se présenter comme vision du monde ? Voilà donc comment le positivisme conçoit le trou qu'il lui reste à remplir ; et comme il avait déjà, dans le cadre de son développement ordinaire et séculaire, très raisonnablement accompli les sept huitièmes de ce programme, on aurait pu croire qu'il suffisait d'un petit bond en avant pour fermer la question. Mais en fait, un peu comme si ayant terminé d'assembler un moteur nous découvrions qu'il reste un piston à mettre en place, au regard de cette ultime question tout l'acquis du passé s'est dissipé dans le néant et le positivisme fut abandonné à une gigantesque révolution ; il lui 
fallait repartir à zéro sur de nouvelles fondations, réanalyser les faits et réorganiser le savoir ; il lui fallait, par exemple, fonder sur une logique ambivalente une science indéterministe de l'incertitude fondamentale du désordre réel ; il lui fallait, par exemple encore, transverser la conscience dans l'électron, la liberté dans la matière, la déviance dans la molécule, la volonté dans les gènes ou le sens dans l'organe à sens. Bref, ayant eu le courage de son but, il lui fallait avoir l'audace de ses moyens et la finesse de ses apparences (i.e. la finesse d'accomplir le tout sous la légitimité de l'antique science). Or, chacun peut le constater, les solutions passent de plus en plus rapidement et l'exigence synthétique demeure; les recommencements de la science jouissent un temps de l'effet de nouveauté, vieillissent subitement et prennent place dans l'histoire des doctrines de leur vivant. À la limite, les scienza nuova, les nouvelles synthèses et les nouveaux paradigmes ne pourront plus se succéder assez rapidement pour tenir à jour la vision globale du puzzle des sciences et ce dernier, constamment augmenté de toutes ces nouvelles sciences, aura toujours plusieurs spécialités d'avance sur la vision globale. Et ainsi nous pourrons nous souvenir que c'est le jour où nous avons désiré un remède au caractère dissolu du savoir scientifique (remède qui aurait été la solution finale de la pensée) que la dissolution définitive de la science nous fut offerte.

En attendant que l'inflation copernicienne galope jusqu'au bout de sa démesure et que prenne fin l'époque des grosses coupures et autres ruptures, revenons à notre épopée et essayons de déterminer en quoi la conception de l'exigence synthétique ici incriminée porte à faux. D'abord, et pour prendre les choses simplement, subordonner la possibilité d'une vision du monde à la possibilité d'une vision globale du puzzle des sciences est une opération étrange : quelle que soit l'ambiguïté de la notion de "vision du monde », on s'entend en général pour désigner là une chose que les ignorants mêmes se payent et sans demander la charité aux "encyclo-pédants». Il est même probable qu'étant imperméable à tout savoir ou pseudo-savoir susceptibles de disqualifier l'expérience quotidienne élémentaire, la vision du monde des premiers soit plus colorée quant à son contenu et plus complexe quant à ses modalités d'intégration. De toutes manières, et même en laissant flotter la notion dans le vague où nous la trouvons ici, l'identification de la vision du monde à une vision globale du puzzle des 
sciences est un coup de force anti-démocratique : pas de vision du monde pour ceux qui lisent des photos-romans dans le métro ou même qui Expérimentent le grand amour ; ni pour ceux qui sont à la Recherche d'un emploi, qui Observent les us et coutumes ou qui Concluent une bonne affaire. Le domaine de ce qui est ici intitulé « vision du monde et de l'homme » nous est ainsi donné pour être la science, cette chose au sein de laquelle, sous nos yeux, Pie est peut-être en train de déblayer un terrain pour sa "philosophie » de la vie. C'est bien d'un changement de terrain qu'il s'agit mais d'un changement parfaitement arbitraire qui dissout la notion même d'expérience (sociale/ historique par définition) en déménageant celle-ci sur le terrain de la science pour ce qui est de son éventuelle intégration. Cette opération nous donne d'ailleurs une première idée de ce "monde " que la nouvelle synthèse a en vue : il s'agit évidemment du monde réel, c'est-à-dire celui dont les différentes sciences se sont approprié (sur le mode cognitif) les différentes parties. Synthétisez ces connaissances dispersées, professe-t-elle, et une vision du monde vous aurez. Autrement dit, ce qu'expérimentent par bouts les disciplines est la totalité du réel (dont le moins qu'on puisse dire c'est qu'il englobe l'homme et le monde), et toutes les modalités indisciplinées de l'expérience ne peuvent que retomber sur ce réel-là, mais sans s'en rendre compte et sans en prendre connaissance. L'expérience non scientifique étant ainsi posée comme non-expérience, il est normal de penser ensuite que ses « visions » doivent lui être inculquées de l'extérieur.

\section{... du seul sujet.}

$\underline{\text { Retour à la table des matières }}$

Il est certain que l'accumulation des connaissances scientifiques dont nous parlions plus haut, la prolifération des sciences et l'extension de leur domaine d'objectivité dessinent ensemble un mouvement historique d'inscription de pratiques sociales dans les schémas de l'activité scientifique, mouvement dont la théorie reste à l'état sauvage sous la topique de "révolution scientifique et technique "; or, ce mouvement, le positivisme le dédaigne en tant que tel pour le saisir plutôt, conformément à son désir, dans son prolongement formel, c'est-à-dire sous la forme de cette limite très « théorique » où rien n'est 
plus un objet qui n'est pas d'abord objet de science. Quant au « Je », tout aussi étiré vers sa limite, qui dans cette projection doit se montrer capable d'expérimenter « le » monde, il est déjà (bien qu'il soit devenu impossible de l'appeler par son nom) une position déterminée de ce système où le "seul objet » (c'est-à-dire l'opération de raturer tout procès historique d'objectivation mais d'en retenir l'horizon comme substance) appelle en face de lui ce pur espace d'accumulation de la connaissance, son seul sujet ; un seul objet, donc une seule science.

De là se commande le projet synthétique : la science, en effet, est morcelée, dispersée, multiple et hétérogène; le sujet universel de l'unique objet est schizophrène et encore inconscient de lui-même car chacun des supports du processus scientifique est assigné par la logique cumulative à une place déterminée dans un ensemble dont l'unité, dès lors, ne se réfléchit nulle part et peut seulement, dans un premier temps, être déduite de l'unité a priori de l'unique objet. La science, en conséquence, de par la dispersion de son contenu dans les sciences se détermine d'abord seulement comme le lieu vide d'une unité virtuelle, comme le point focal où se rencontrent les prolongements imaginaires de l'ensemble des disciplines, chacune à la poursuite d'une facette donnée du seul objet. Il s'agit donc pour le synthétiseur de fonder toutes les sciences en les rapportant systématiquement les unes aux autres et de laisser se déduire de leurs combinaisons le discours propre à habiter, au centre, la place encore vide du sujet virtuel de tout rapport à l'unique objet. La science, accédant alors en tant que science synthétique à la plénitude de son contenu et récupérant l'ensemble des résultats accumulés à son compte, pourra enfin se poser elle-même comme sujet actuel du seul objet, c'est-à-dire comme unité réfléchie de la totalité de l'expérience du monde.

En ce sens, à son point de vue à tout le moins, la nouvelle synthèse est bien autre chose que la constitution d'un autre «body of knowledge »; elle est plutôt l'orgie théorique exhaustive dont peut seule procéder la formation of «the » body of knowledge. Cependant, si le puzzle des sciences désigne négativement le lieu où Pic doit placer son cerveau pour l'offrir comme support à ce procès de formation, ce n'est pas qu'il ambitionne de devenir lui-même le sujet; la science synthétique du réel exige au contraire de l'individu une modestie tout hégélienne et si la combinaison des sciences-membres et le processus 
de formation du corps de la connaissance a besoin d'un cerveau-utérus (c'est-à-dire d'un contenant infrathéorique naïf mais déjà homogène et unifié par nature) ainsi que d'un témoin (c'est-à-dire d'un individu en tant que conscience supra-théorique de la science qui se combine en lui indépendamment de lui), elle se passe radicalement d'un docteur Frankestein qui prendrait personnellement l'affaire en main et qui imposerait au résultat l'allure de sa propre fantaisie. En toute rigueur, l'accession du sujet universel à la conscience de soi au travers de la tête de Pic doit être pensée selon le modèle bio-chimique : elle est la synthèse, dans l'éprouvette mirandolienne, du corps de la connaissance à partir de ses constituants et le rôle de l'individu se limite à fournir une éprouvette vide, à la placer dans la vallée du puzzle, à laisser s'y déverser, ruisselante ou torrentielle, toute expérience scientifique qui peut être faite des flancs du seul objet et à témoigner du résultat. $\mathrm{Si}$, donc, le synthétiseur parle de lui-même, s'il confesse ses défaillances ou son bonheur, s'il nomme en préface les cent cinquante amis intimes qu'il s'accuse d'avoir exploité dans une relation à sens unique, ce n'est pas parce qu'il veut occuper personnellement la place du Sujet ; c'est plutôt pour se libérer de sa subjectivité en la rendant publique et montrer qu'il est capable de tenir son particulier à distance du processus auquel il a prêté sa tête car, étant en quelque sorte le concierge de celle-ci, il est de son devoir de la tenir propre. Ainsi, lorsqu'il dit par exemple : « J'essaie de structurer mon plan » et signale les maux de tête et les angoisses qui accompagnent cet effort, c'est pour se donner aussitôt l'occasion d'une distinction selon laquelle le support, même souffrant, ne doit pas se prendre pour le sujet : « J'essaie de structurer mon plan. Je ?... C'est la pensée, la puissance organisatrice qui cherche à faire ses combinaisons, ses associations, ses assemblages à travers moi... " ${ }^{6}$ Bref, la connaissance ne se laisse plus saisir selon le schéma général de l'activité mais tout au plus selon celui de la passivité ; certaines circonstances étant réunies, elle est quelque chose qui se produit dans le réel, une sorte de maladie agréable du cerveau. Et comme cela fait partie du réel au même titre que le reste, la science synthétique du réel ne pourra pas éviter de se terminer sur une théorie de la connaissance du réel, théorie qui s'élèvera ainsi enfin au rang de simple et transparente conscience de soi de la science. De cette manière, le synthétiseur pourra faire disparaître le crédit qu'il

6 Edgar Morin, Le Journal de Californie, Éditions du Seuil, Paris, 1970, p. 228. 
s'est avancé au début et saturer l'espace de son jeu de mot inaugural ; ayant été le lieu de la connaissance acquise par la nature de la nature, il pourra rembourser sa dette et verser au compte de la nature la connaissance de la connaissance ; il aura donc comblé définitivement le trou du sujet et en aura fait disparaître l'ouverture en même temps que le déficit : alors seulement pourra-t-il, en tant que physis, être auprès de lui-même, sans détour.

Gilles Gagné, Département de sociologie, Université Laval 